\title{
Rotating helical turbulence. II. Intermittency, scale invariance, and structures
}

\author{
P. D. Mininni ${ }^{1,2}$ and A. Pouquet ${ }^{2,3}$ \\ ${ }^{1}$ Departamento de Física, Facultad de Ciencias Exactas y Naturales, Universidad de Buenos Aires \\ and CONICET, Ciudad Universitaria, 1428 Buenos Aires, Argentina \\ ${ }^{2}$ Computational and Information Systems Laboratory, NCAR, P.O. Box 3000, Boulder, \\ Colorado 80307-3000, USA \\ ${ }^{3}$ Earth and Sun Systems Laboratory, NCAR, P.O. Box 3000, Boulder, Colorado 80307-3000, USA
}

(Received 7 September 2009; accepted 6 February 2010; published online 24 March 2010)

\begin{abstract}
We study the intermittency properties of the energy and helicity cascades in two $1536^{3}$ direct numerical simulations of helical rotating turbulence. Symmetric and antisymmetric velocity increments are examined, as well as probability density functions of the velocity field and of the helicity density. It is found that the direct cascade of energy to small scales is scale invariant and nonintermittent, whereas the direct cascade of helicity is highly intermittent. Furthermore, the study of structure functions of different orders allows us to identify a recovery of isotropy of strong events at very small scales in the flow. Finally, we observe the juxtaposition in space of strong laminar and persistent helical columns next to time-varying vortex tangles, the former being associated with the self-similarity of energy and the latter with the intermittency of helicity. (C) 2010 American Institute of Physics. [doi:10.1063/1.3358471]
\end{abstract}

\section{INTRODUCTION}

Turbulence is often referred to as the last unresolved main problem of classical physics. The diversity of applications of turbulent flows, from geophysics and astrophysics to engineering, and the observed complexity and lack of predictability of such flows both make the problem difficult to tackle, or even to define. Part of this complexity is due to the fact that turbulence comes in intermittent "gusts," strong events that are scarce, but still more frequent that what could be expected if normally distributed. These gusts give rise to the well-known breakdown of scale invariance in the flow. ${ }^{1}$

Intermittency is a highly spatially and temporally localized phenomenon. It is believed to be associated only with a forward cascade of an ideal invariant (a quantity conserved in the inviscid case), corresponding to the transfer of this quantity toward small scales with constant flux as a result of the nonlinear coupling between modes; it is not directly related with the dimensionality of the problem. As an example, the energy cascade from larger to smaller scales in isotropic and homogeneous three dimensional turbulence is intermittent. ${ }^{2}$ Magnetofluids, i.e., conducting fluids where the velocity field is coupled to the magnetic field, are intermittent in two ${ }^{3}$ and in three dimensions, ${ }^{4}$ and in both cases a direct cascade of energy takes place. These examples are in contrast with the two dimensional neutral fluid case, for which the conservation of vorticity leads to an inverse energy cascade (a transfer of energy to the large scales with constant flux). While the direct cascade of enstrophy in this case is intermittent, there is evidence that the inverse cascade of energy is scale invariant and probability density functions (PDFs) of velocity fluctuations are close to Gaussian. ${ }^{5}$ For the case of rotating turbulence, studies of intermittency are not numerous since they require high Reynolds numbers together with low Rossby numbers; moreover, since in this case energy undergoes both a direct and an inverse cascade, ${ }^{6-8}$ it is unclear a priori whether intermittency is to be expected in the small scales or not, although, until the present work (see Sec. IV), there was no documented example of self-similar direct energy cascade to our knowledge. In the experiments of freely decaying rotating turbulence, ${ }^{9}$ it was found that rotation significantly decreases intermittency, although strictly scale-invariant (nonintermittent) statistics were not found. Such experimental results are also in agreement with recent analyses of data stemming from direct numerical simulations (DNS) of nonhelical rotating turbulence. ${ }^{10,11}$ Other laboratory experiments ${ }^{12}$ reported an even more pronounced reduction of the intermittency when rotation is present.

Although intermittency is believed to take place at small scales, strong events can affect the dynamics of the large scales, especially in systems close to criticality. As an example, it was shown in Refs. 13-15 that local fluctuations of the kinetic helicity $H_{V}=\mathbf{u} \cdot \nabla \times \mathbf{u}$ with $\mathbf{u}$ the velocity can explain phase and amplitude variations of the 22-years solar cycle. Also, intermittency is known to affect the transport of momentum in atmospheric surface layers. ${ }^{16}$

Considering the large number of degrees of freedom typical in turbulence (typical mesoscale flows in the atmosphere of the Earth can have, for instance, about $10^{18}$ degrees of freedom), many of the approaches to turbulence rely on an assumed scale invariance to model the flow statistics at small (often unresolved) scales. The search for self-similar quantities in three-dimensional turbulence is a long-standing problem, which would relate its study with critical phenomena and the out-of-equilibrium statistics of systems with a large number of modes, and which would allow the use of tools from quantum field theory, condensed matter, and statistical mechanics. 
In a previous paper, hereafter referred to as Paper I, ${ }^{17}$ we presented results from two massive numerical simulations of helical rotating turbulence. Rotation and helicity are present in many atmospheric phenomena, as e.g., supercell storms and tornadoes. ${ }^{18-20}$ In the simulations, instead of having physical effects that inject helicity in the flow as, e.g., interplay between rotation and stratification or rotating flows near solid boundaries, we used a helical body force. In spite of the simplification, this allows for savings in computing time while allowing us to focus solely on the effect the presence of helicity has in rotating turbulence. The simulations confirmed the scaling laws for the energy and helicity spectra predicted in Ref. 21. The development of anisotropies in the flow as well as scaling laws in the directions parallel and perpendicular to the axis of rotation was also studied, considering both global measurements of anisotropy as well as spectral quantities.

In this paper we study the intermittent properties of both the direct cascade of energy and the direct cascade of helicity in helical rotating turbulence, using the data from the simulations presented in Paper I. A decomposition into directions parallel and perpendicular to the axis of rotation is used to study anisotropy. Details of this decomposition, as well as the definitions of increments, structure functions, and scaling exponents used to quantify the intermittency, are given in Sec. II. The results of the decomposition performed on the data stemming from the high resolution DNS are presented in Sec. III, where the possible recovery of isotropy at small scales is discussed. Averaging over different directions and over time, up to $4.3 \times 10^{11}$ data points were used to obtain statistical evidence that the direct cascade of energy is scale invariant, while the direct cascade of helicity is not, as discussed in Sec. IV. This result is obtained from the study of structure functions as well as PDFs of velocity and helicity increments (Sec. V). Finally, the development of structures at large and small scales in the flow is discussed in Sec. VI and the conclusions are presented in Sec. VII.

\section{VELOCITY AND HELICITY INCREMENTS}

\section{A. Increments}

To study intermittency in the direct cascade of energy, we will use longitudinal increments of the velocity field $\mathbf{u}$,

$$
\delta u(\mathbf{x}, \ell)=[\mathbf{u}(\mathbf{x}+\ell)-\mathbf{u}(\mathbf{x})] \cdot \frac{\ell}{|\ell|},
$$

where the increment $\ell$ can be in any direction. Structure functions of order $p$ are then defined as

$$
S_{p}(\ell)=\left\langle\delta u^{p}(\mathbf{x}, \ell)\right\rangle,
$$

where the brackets denote spatial average over all values of $\mathbf{x}$. The structure functions depend on the direction of the increment, and no assumption about isotropy or axisymmetry has yet been made.

In the following, we briefly discuss the motivations to use structure functions, and mention some well-known results for isotropic and homogeneous flows. We clearly state which results hold only for isotropic flows, and which expressions are independent of this assumption. In the latter case, the expressions will be specialized for the axisymmetric case considering increments parallel and perpendicular to the axis of rotation, as described in the next subsection. The development of anisotropy in rotating flows can also be studied using, e.g., the dimensionality structure tensor. ${ }^{22}$

The $S_{p}$ structure functions are of interest because for $p=2$ they are related to the two-point correlation function of the velocity, and thus to the energy spectrum through the convolution theorem. Therefore, a power law behavior can be expected for scales corresponding to the inertial range. Also, for the isotropic and homogeneous case, $S_{3}$ is related to the energy flux and scales linearly with the increment $\ell$ in the inertial range. ${ }^{23}$ As a result, if the direct cascade is scale invariant, for an isotropic and homogeneous flow $S_{p}(\ell) \sim \ell^{p / 3}$, and departures of the exponents from this relation are a signature of intermittency.

The study of intermittency in the direct cascade of helicity has been less explored. In the isotropic and homogeneous case, one can study it using for example structure functions based on the helicity flux, which is a third order field. ${ }^{24,25}$ In real space, this flux can be written in two different ways, which follow from the right-hand side of the KármánHowarth theorem for the helicity, ${ }^{26,27}$

$$
\left\langle\left[\mathbf{u}(\mathbf{x}) \cdot \frac{\ell}{|\ell|}\right]\left[(\mathbf{u}(\mathbf{x}) \times \mathbf{u}(\mathbf{x}+\ell)) \cdot \frac{\ell}{|\ell|}\right]\right\rangle=\frac{1}{15} \widetilde{\varepsilon} \ell^{2},
$$

or in terms of structure functions of the velocity and vorticity, $^{28}$

$$
\begin{aligned}
& \langle\delta u(\mathbf{x}, \ell)[\delta \mathbf{u}(\mathbf{x}, \ell) \cdot \delta \boldsymbol{\omega}(\mathbf{x}, \ell)]\rangle-\frac{1}{2}\left\langle\delta \omega(\mathbf{x}, \ell)(\delta \mathbf{u}(\mathbf{x}, \ell))^{2}\right\rangle \\
& \quad=-\frac{4}{3} \widetilde{\varepsilon} \ell
\end{aligned}
$$

where $\widetilde{\varepsilon}$ is the helicity injection rate. Both relations are equivalent, as it is easy to see from $\delta \omega \sim \delta u / \ell .{ }^{28}$ However, here we want structure functions that can be associated to the spectral scaling, and must therefore be based on second order quantities. There are two candidates that follow from the expressions in the time derivative appearing in the KármánHowarth theorem for helicity, ${ }^{26,27}$

$$
\left\langle\left[u_{i}(\mathbf{x}+\ell)-u_{i}(\mathbf{x})\right]\left[u_{j}(\mathbf{x}+\ell)-u_{j}(\mathbf{x})\right]\right\rangle,
$$

or $^{28}$

$$
\langle\delta \mathbf{u}(\mathbf{x}, \ell) \cdot \delta \boldsymbol{\omega}(\mathbf{x}, \ell)\rangle .
$$

As written here, these structure functions depend on the direction of the increment and therefore isotropy or axisymmetry has not been assumed yet. Both quantities are Galilean invariant, as is required for the structure functions to be well behaved. ${ }^{29}$ The first quantity is a second order tensor with indices $i, j$, while the second is a scalar. For simplicity, here we use the second expression, although both are related to the antisymmetric part of the two-point correlation tensor for the velocity. Then, structure functions for the helicity (without assuming isotropy) can be defined as

$$
\mathcal{H}_{p}(\ell)=\left\langle[\delta \mathbf{u}(\mathbf{x}, \ell) \cdot \delta \boldsymbol{\omega}(\mathbf{x}, \ell)]^{p}\right\rangle .
$$

With this definition, for isotropic and homogeneous 
turbulence the assumption of scale invariance leads to $\mathcal{H}_{p}(\ell) \sim \ell^{p / 3}$. Note that $\mathcal{H}_{1}$ is second order in the velocity whereas $S_{1}$ is first order.

\section{B. Parallel and perpendicular directions}

The development of anisotropies in a rotating flow has been studied in experiments ${ }^{30-32}$ and in numerical simulations (see e.g., Ref. 33). Anisotropy was shown to develop in a range of Rossby numbers such that nonlinear interactions are not completely damped with the scrambling effect of inertial waves. ${ }^{34}$ The presence of rotation breaks down the isotropy of the flow, introducing a preferred direction. Energy, as a result of resonant triad interactions, is transferred preferentially toward modes in spectral space perpendicular to the axis of rotation, ${ }^{6,734}$ although linear effects may also be relevant in the formation of the structures. ${ }^{35}$

In isotropic and homogeneous turbulence, it is a common practice to study velocity increments (or two-point correlation functions) in only a few directions, or to average over different directions as in, e.g., the leading (isotropic) term of the $\mathrm{SO}(3)$ decomposition. ${ }^{36,37}$ Given the preferred direction in our problem and the natural axisymmetry associated with it, we will be interested in increments parallel and perpendicular to the angular rotation $\boldsymbol{\Omega}$, which will be denoted, respectively, as $\ell_{\|}$and $\ell_{\perp}$. In principle, in the plane perpendicular to $\boldsymbol{\Omega}$, the increments $\ell_{\perp}$ can be taken in every possible direction and later averaged to obtain structure functions that only depend on the scalar increment $\ell_{\perp}$. However, this requires interpolation of the three components of the velocity in the three dimensional space every time an increment does not reside on a computational grid point. To avoid the computational cost of this interpolation, we follow the procedure introduced in Ref. 38 for the isotropic case. We only compute increments given by the product of an integer times a generator vector, with the generator vectors chosen such that they fill as uniformly as possible circles in the plane perpendicular to $\boldsymbol{\Omega}$, and such that their product with an integer always falls on a grid point.

Twelve generators were used in the $x-y$ plane: $(1,0,0)$, $(1,1,0), \quad(2,1,0),(3,1,0),(0,1,0),(-1,1,0),(1,2,0),(-2,1,0)$, $(-1,2,0),(1,3,0),(-3,1,0)$, and $(-1,3,0)$ (in units of grid points in the simulation). These generators, plus the 12 generators obtained by multiplying them by -1 (or equivalently, considering negative increments) cover the plane in an approximately uniform way (see Fig. 1). In the $z$ direction (the direction parallel to the axis of rotation) the generator for the increments is the vector $(0,0,1)$. Given these generators, increments in Eqs. (2) and (7) are created by multiplying the generators by integer numbers. With these choices, all increments reside on grid points and no interpolation in the computation of the structure functions for each direction is required. Once structure functions for all directions have been computed, structure functions in the perpendicular direction $S_{p}\left(\ell_{\perp}\right)$ and $\mathcal{H}_{p}\left(\ell_{\perp}\right)$ are obtained by averaging over the results for the 12 directions in the $x-y$ plane. As the generators have different lengths, interpolation in this step is required, but it is less costly from the computational point of view as only interpolation of scalar one-dimensional functions is

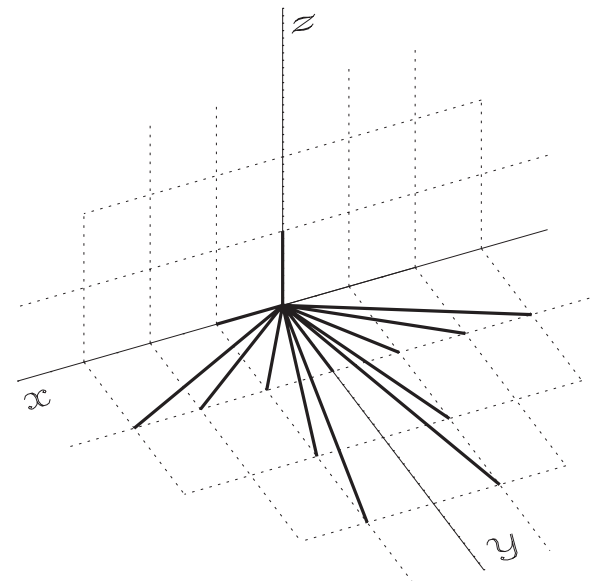

FIG. 1. The 12 generators used to compute increments in the $x-y$ plane and the generator in the $z$ direction. The crossings of dotted lines indicate grid points in the numerical simulation.

needed. The structure functions in the parallel direction, $S_{p}\left(\ell_{\|}\right)$and $\mathcal{H}_{p}\left(\ell_{\|}\right)$, are obtained directly from the generator in the $z$ direction. Finally, average in time (using snapshots of the velocity field at different turnover times) can be computed.

In our case, we use for the run with the larger rotation rate, $(\Omega=9)$, ten snapshots of the velocity field spanning ten turnover times, from $t=20$ to 30 (see Paper I). As a result, considering that each snapshot has $1536^{3} \approx 3.6 \times 10^{9}$ grid points, and considering the twelve generators used, each increment in $S_{p}\left(\ell_{\perp}\right)$ and $\mathcal{H}_{p}\left(\ell_{\perp}\right)$ results from an averaging over $4.3 \times 10^{11}$ data points. In the case of $S_{p}\left(\ell_{\|}\right)$and $\mathcal{H}_{p}\left(\ell_{\|}\right)$, each increment is obtained using $\approx 3.6 \times 10^{10}$ data points.

\section{STRUCTURE FUNCTIONS}

\section{A. Velocity structure functions}

Two simulations were used for the analysis, described in more detail in Paper I. One of the simulations (hereafter, run A), has $\Omega=0.06$, a Rossby number Ro $\approx 8.5$, and was continued for almost ten turnover times. The other simulation (run B) has $\Omega=9$, a Rossby number Ro $\approx 0.06$, and was continued for 30 turnover times. Both runs have a Reynolds number $\operatorname{Re} \approx 5100$.

Figure 2 shows the result of computing the velocity structure functions in all directions for one snapshot of the field (at $t=30$ in run B), and of averaging over the different directions to obtain $S_{2}\left(\ell_{\perp}\right)$ and $S_{2}\left(\ell_{\|}\right)$. At small scales, both structure functions scale as $\ell^{2}$, as can be expected for a wellresolved smooth flow in the dissipative range. At intermediate scales, an inertial range with power law scaling can be identified in $S_{2}\left(\ell_{\perp}\right)$, but not in $S_{2}\left(\ell_{\|}\right)$. Indeed, $S_{2}\left(\ell_{\|}\right)$is smaller than $S_{2}\left(\ell_{\perp}\right)$ at all scales (especially so at the largest scales), and shows no clear scaling. This is consistent with the results obtained in Paper I from the energy spectrum: while the energy spectrum in perpendicular wave vectors shows an inertial range with power law behavior and approximately constant flux, the energy spectrum in the parallel direction shows no clear scaling and its associated flux decays rapidly with scale. 


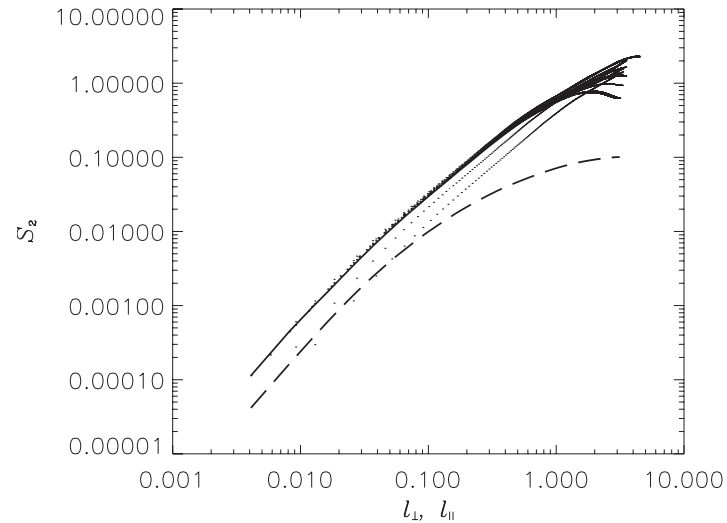

FIG. 2. Second order structure functions $S_{2}$ at $t=30$ in run B with $\mathrm{Ro}=0.06$. The dotted lines indicate the different structure functions in the 12 directions given by the generators in the $x-y$ plane, and the thick solid curve is the average $S_{2}\left(\ell_{\perp}\right)$. The thick dashed curve corresponds to increments in the $z$ direction and is $S_{2}\left(\ell_{\|}\right)$.

Figure 3 shows the structure functions $S_{2}\left(\ell_{\perp}\right)$ and $S_{2}\left(\ell_{\|}\right)$ at four different times between $t=20$ and 30 in run B. No substantial differences are observed at the different times. The average scaling exponent in the inertial range $S_{2}\left(\ell_{\perp}\right)$ $\sim \ell_{\perp}^{\zeta_{2}}$, obtained after averaging over the ten turnover times, is $\zeta_{2}=1.41 \pm 0.02$, and is indicated in the figure by the straight line. Errors here and in the following are defined as the standard mean error

$$
e_{\zeta_{p}}=\frac{1}{N} \sqrt{\sum_{i=1}^{N}\left(\zeta_{p i}-\overline{\zeta_{p}}\right)^{2}},
$$

where $\zeta_{p i}$ is the slope obtained from a least square fit for each snapshot $i, N$ is the number of snapshots, and $\bar{\zeta}_{p}$ is the mean value averaged over all snapshots. The error in the least square determination of the slope for each snapshot is much smaller than this standard mean error for the averaged exponent. Extended self-similarity is not used to obtain the slopes.

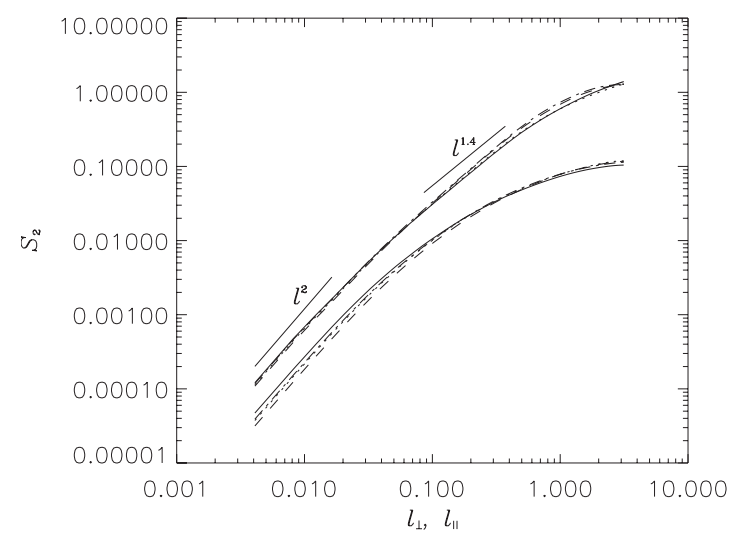

FIG. 3. Second order structure functions $S_{2}\left(\ell_{\perp}\right)$ and $S_{2}\left(\ell_{\|}\right)$in run B at different times, between $t=20$ and 30. A dissipative range scaling $\sim \ell^{2}$ is indicated at small scales, and the average slope $\zeta_{2} \sim 1.41$ is indicated in the inertial range.

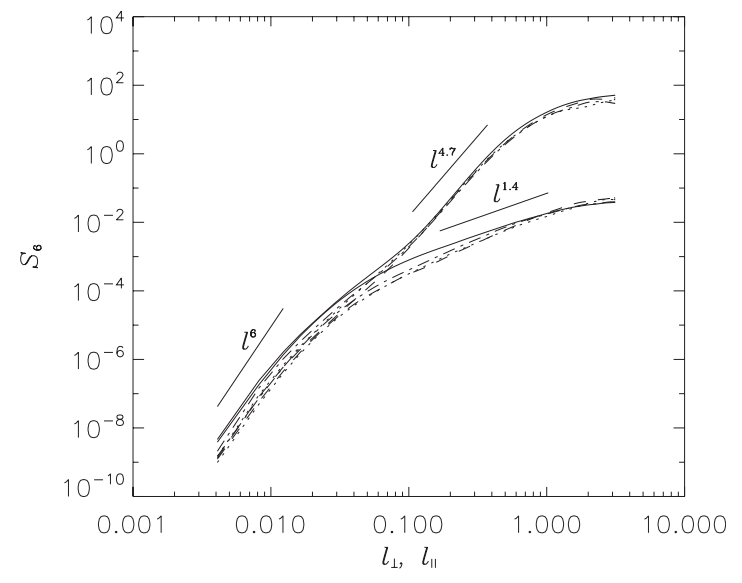

FIG. 4. Sixth order structure functions $S_{6}\left(\ell_{\perp}\right)$ and $S_{6}\left(\ell_{\|}\right)$in run B at different times, between $t=20$ and 30. A dissipative range scaling $\sim \ell^{6}$ is indicated at small scales, and two average slopes are indicated in the inertial ranges (see text). Note that the perpendicular part of the structure function dominates the parallel one at all scales.

The value obtained for $\zeta_{2}$ is in good agreement with predictions for helical rotating turbulence. ${ }^{21}$ In a rotating flow with maximal helicity, $E \sim k^{-2.5}$, which leads to $S_{2}\left(\ell_{\perp}\right) \sim \ell_{\perp}^{1.5}$. For flows with nonmaximal helicity the $\zeta_{2}$ exponent is, according to Ref. 21, between 1 and 1.5, with the value of 1 corresponding to the nonhelical case. Note that in numerical simulations of nonhelical rotating turbulence $S_{2}\left(\ell_{\perp}\right) \sim \ell_{\perp}$ was reported in Refs. 10 and 11 .

From the behavior of the amplitude of $S_{2}\left(\ell_{\|}\right)$with scale, it seems isotropy could be recovered at small scales in a rotating flow if the inertial range is wide enough for $S_{2}\left(\ell_{\|}\right)$ and $S_{2}\left(\ell_{\perp}\right)$ to collapse (or, in other words, for the effect of rotation to be negligible at the smallest scales). Indeed, for small values of $\ell$ the parallel and perpendicular structure functions become closer. This is more evident in velocity structure functions of higher order. As an example, Fig. 4 shows the sixth order velocity structure function. While an inertial range in the perpendicular direction is still visible (the mean slope averaged over ten turnover times is indicated as a reference), and the structure functions in this direction do not change much between different snapshots, such is not the case at smaller scales or in the parallel direction. In the parallel direction, larger fluctuations between different times are observed. Moreover, the anisotropic inertial range is shortened as, at small scales, both $S_{6}\left(\ell_{\perp}\right)$ and $S_{6}\left(\ell_{\|}\right)$collapse to a single curve. This collapse takes place for scales smaller than $\ell \approx 0.05$, with slight fluctuations in time; it indicates that the strongest events in the flow (which begin to dominate structure functions as the order increases) tend toward isotropy at the smallest scales. Note that the collapse is not observed in the $S_{2}$ structure functions (see Fig. 3), whereas $S_{p}\left(\ell_{\perp}\right)$ and $S_{p}\left(\ell_{\|}\right)$get closer to each other at smaller scales as the order $p$ is increased.

This partial recovery of isotropy in the higher order moments of the velocity field can be understood as follows: higher values of $p$ in Eq. (2) leave only the strongest gradients contributing to $S_{p}\left(\ell_{\perp}\right)$ and $S_{p}\left(\ell_{\|}\right)$, as the contribution of mild gradients to the structure functions goes to zero as $p$ is increased. It is only these strong events at small scales that 
are more isotropically distributed, as indicated by Fig. 4 (at least at the Reynolds numbers considered here). However, mild gradients are still anisotropic at small scales, as follows from Fig. 3. Simulations with more spatial resolution at larger Reynolds numbers will be required to study if this transition toward isotropy only takes place for the strong events, or whether for sufficiently small scales the transition takes place for all orders, with a change in the spectral index of the flow (see e.g., Refs. 39-41 for phenomenological treatments of nonhelical rotating turbulence that consider the possibility of such a transition).

Similarly to the stratified case, one can introduce a wave number at which rotation and nonlinear advection balance (see Refs. 39 and 40),

$$
k_{\Omega}=\left(\Omega^{3} / \epsilon\right)^{1 / 2}
$$

(with $\epsilon$ the energy dissipation rate); $k_{\Omega}$ can be considered as the largest wave number where rotation effects are important; the inverse of this wave number, $\ell_{\Omega}=2 \pi / k_{\Omega}$, is equivalent to the Ozmidov length in stratified turbulence, which separates the inertial range (at smaller scales) from the range dominated by buoyancy (at larger scales). It is rather remarkable that in our simulation $\ell_{\Omega} \approx 0.04$, close to the value of $\ell \approx 0.05$ where the transition in $S_{p}\left(\ell_{\perp}\right)$ takes place for large values of $p$ (see Fig. 4). However, a confirmation of this would require a parametric study varying the value of $\Omega$, which in DNS at the spatial resolution considered here is out of reach with present day computers.

At this point, a discussion about units is in order. The distance between grid points in our runs is $2 \pi / 1536$ $\approx 0.004$. This is also the smallest distance for which increments can be computed in the structure functions. Since the simulations are de-aliased using the $2 / 3$ rule, the largest wave number resolved is $k_{\max }=512$, which corresponds to a length $\ell_{\min }=2 \pi / k_{\max } \approx 0.01$. As a result, the velocity field at scales between $\approx 0.004$ and $\approx 0.01$ must be necessarily smooth, and its structure functions should scale as $S_{p} \sim \ell^{p}$ as it is indeed the case (see e.g., Fig. 3). The dissipation scale in the simulations is just slightly larger than $\ell_{\min }$, which explains why the $\sim \ell^{p}$ scaling extends a little bit beyond $\ell_{\min }$ (as required for the simulations to be well resolved). For practical purposes, we can estimate the dissipation scale to be between $\approx 0.01$ and 0.02 (these values are consistent with estimations from the energy spectrum, shown in Paper I). Since the collapse of the parallel and perpendicular structure functions occurs near $\ell \approx 0.05$, one may wonder if this scale is well resolved, or if the collapse results from numerical cutoff or viscous effects. We computed structure functions for simulations of forced helical and nonhelical rotating turbulence at resolutions of $512^{3}$ grid points (see Refs. 11 and 21) for which $k_{\Omega}>k_{\max }$, and in that case no bump, collapse, or changes in the behavior of the structure functions at the smallest scales were observed. However, simulations at larger resolutions would be desirable to further confirm this result.

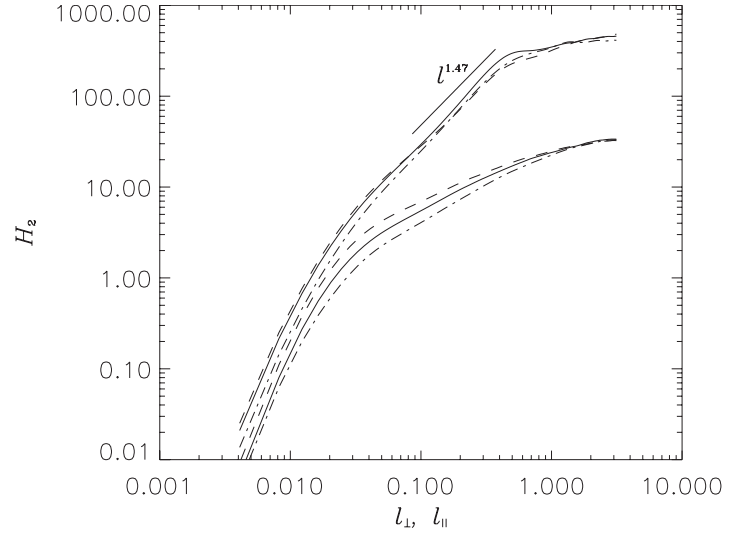

FIG. 5. Second-order helicity structure functions $H_{2}\left(\ell_{\perp}\right)$ and $H_{2}\left(\ell_{\|}\right)$(see Eq. (7)) in run B with Ro=0.06. at different times between $t=20$ and 30. The dissipative range scales as $\sim \ell^{4}$, consistent with the fact that $H_{2}$ is quartic in the velocity; the average slope is indicated for the inertial range.

\section{B. Helicity structure functions}

Helicity structure functions are computed in the same fashion as velocity structure functions [see Eq. (7)]; the functions $H_{2}\left(\ell_{\perp}\right)$ and $H_{2}\left(\ell_{\|}\right)$for different times are shown in Fig. 5 , after averaging in the different directions. It should be noted that the $\mathrm{H}_{2}$ structure functions are effectively of fourth order in the fields, and as a result the convergence of the statistics is not as good in this case as it was for the structure functions discussed in the previous section for the velocity. Also as a result of the higher-order dependence on the fields, the dissipative range scales as $\ell^{4}$. Moreover, helicity is not a positive definite quantity, and cancellations between regions with positive and negative alignments of the velocity and the vorticity can take place, resulting in larger fluctuations of the increments. Convergence of the statistics for all orders studied here was checked by computing the cumulants for each moment (see e.g., Refs. 42 and 43).

In the case of the helicity structure functions, fluctuations between different temporal snapshots are larger than for the velocity structure functions. This is mostly due to the fact that $H_{2}\left(\ell_{\|}\right)$displays strong fluctuations in time, and that the helicity becomes isotropic at smaller scales more rapidly with increasing $p$. However, fluctuations in the inertial range of the perpendicular increments are smaller, and an inertial range can still be defined. For the second order, defining the scaling exponents as $\mathcal{H}_{p}\left(\ell_{\perp}\right) \sim \ell_{\perp}^{\xi_{p}}$, we obtain $\xi_{2}=1.46 \pm 0.03$. This value leads to a spectral scaling $H(k)$ $\sim k_{\perp}^{-1.7}$ (the prediction for a maximally helical rotating flow is $H(k) \sim k_{\perp}^{-1.5}$ ). The scaling of the energy and of the helicity obtained from the structure functions is consistent with the $e+h=4$ rule for helical rotating flows, where $e$ is the energy spectral index and $h$ is the helicity spectral index $;{ }^{21}$ it is also consistent with the spectral indices measured in Paper I.

Fluctuations become larger for larger values of $p$, and we were unable to compute structure functions for moments with converged cumulants beyond $p=4$ for run $\mathrm{A}$ and $p=5$ for run B for the helicity. Specifically for run B, variations in the amplitude of $\mathcal{H}_{p}\left(\ell_{\|}\right)$increase with $p$, changing the scale where the structure functions become isotropic. As a result, for some snapshots no scaling in the perpendicular direction 


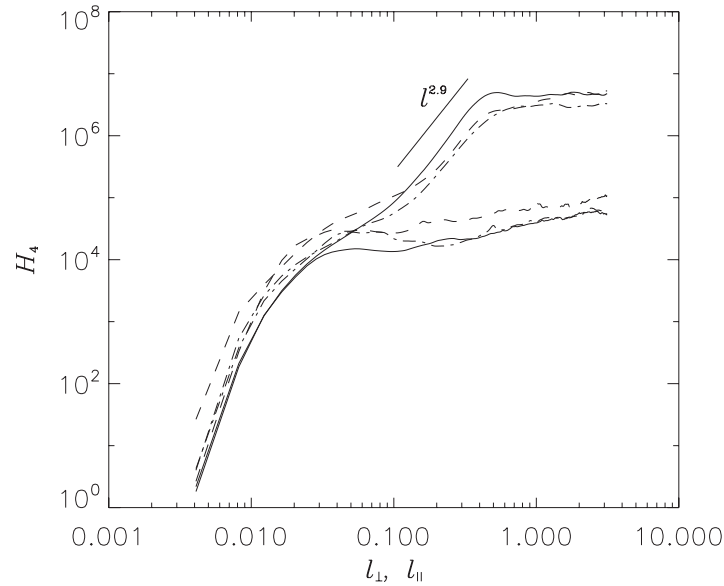

FIG. 6. Fourth-order helicity structure functions $H_{4}\left(\ell_{\perp}\right)$ and $H_{4}\left(\ell_{\|}\right)$in run B at different times, between $t=20$ and 30 . The average slope is indicated for the inertial range.

was observed, and as a rule of thumb snapshots for which $\mathcal{H}_{p}\left(\ell_{\perp}\right)$ and $\mathcal{H}_{p}\left(\ell_{\|}\right)$became of the same order at scales larger than $\ell \approx 0.1$ had to be discarded. Figure 6 shows the fourth order helicity structure functions for four snapshots that present a discernible inertial range in $\ell_{\perp}$ (a total of eight snapshots was used to compute the average scaling exponents).

\section{INTERMITTENCY IN THE DIRECT CASCADES}

In a self-similar flow, scaling exponents depend linearly on the order $p$. As mentioned in Sec. I, the anomalous scaling (the deviation from linear dependence on $p$ ) of the exponents observed in many turbulent flows is a signature of deviations from scale invariance and of intermittency. The velocity and helicity scaling exponents in the direct cascade range of runs $\mathrm{A}$ and $\mathrm{B}$ are shown in Fig. 7.

In run $A$, with $\Omega=0.06$, the effect of rotation is negligible and the velocity scaling exponents display the usual deviation from the Kolmogorov $p / 3$ scaling. Deviations from a straight line are often quantified in terms of the intermittency coefficient $\mu=2 \zeta_{3}-\zeta_{6}$, which for this run is

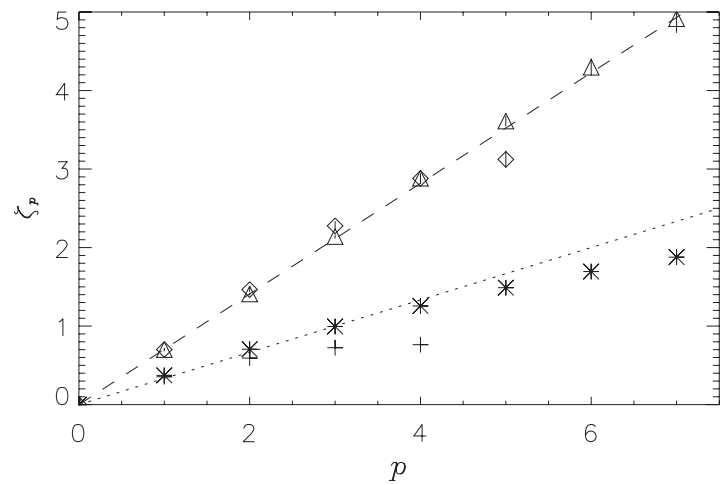

FIG. 7. Scaling exponents (with error bars, see Table I) as a function of the order $p$, for the velocity (stars) and the helicity (pluses) in run A with $\mathrm{Ro}=8.5$, and for the velocity (triangles) and the helicity (diamonds) in run B with $\mathrm{Ro}=0.06$. The dotted line corresponds to Kolmogorov scaling $\zeta_{p}=p / 3$, and the dashed line to $\zeta_{p}=0.71 p$, which represents the velocity exponents best.
TABLE I. Order $p$ and scaling exponents $\zeta_{p}$ for the velocity and $\xi_{p}$ for the helicity, with errors, for run $\mathrm{A}(\mathrm{Ro}=8.5)$ and run $\mathrm{B}(\mathrm{Ro}=0.06)$.

\begin{tabular}{lcccc}
\hline \hline$p$ & $\zeta_{p}$ (run A) & $\xi_{p}$ (Run A) & $\zeta_{p}$ (run B) & $\xi_{p}$ (Run B) \\
\hline 1 & $0.37 \pm 0.01$ & $0.35 \pm 0.01$ & $0.701 \pm 0.007$ & $0.70 \pm 0.03$ \\
2 & $0.70 \pm 0.03$ & $0.59 \pm 0.01$ & $1.41 \pm 0.02$ & $1.46 \pm 0.03$ \\
3 & $0.99 \pm 0.03$ & $0.72 \pm 0.01$ & $2.14 \pm 0.02$ & $2.27 \pm 0.07$ \\
4 & $1.25 \pm 0.04$ & $0.76 \pm 0.02$ & $2.88 \pm 0.04$ & $2.88 \pm 0.07$ \\
5 & $1.49 \pm 0.04$ & $\ldots$ & $3.61 \pm 0.07$ & $3.1 \pm 0.1$ \\
6 & $1.69 \pm 0.05$ & $\ldots$ & $4.3 \pm 0.1$ & $\cdots$ \\
7 & $1.88 \pm 0.05$ & $\ldots$ & $4.9 \pm 0.2$ & $\cdots$ \\
\hline \hline
\end{tabular}

$\mu=0.29 \pm 0.06$, in agreement with previous simulations and experiments of nonrotating turbulence. The third order exponent is $\zeta_{3}=0.99 \pm 0.03$, in good agreement with the value of 1 expected for isotropic and homogeneous turbulence. The higher orders computed in this run are also consistent with results of nonrotating turbulence at very large Reynolds numbers (see e.g., Ref. 43). All values of the scaling exponents up to order eight are given in Table I.

On the other hand, for run B at low Rossby number, the velocity scaling exponents are (within error bars) consistent with a scale invariant (intermittency-free) linear relationship. For this run, $\mu=0.0 \pm 0.1$, compatible with a value of $\mu=0$ which corresponds to a completely scale invariant flow. A similar result was reported in an experimental study ${ }^{12}$ (although associated in that case with a linear scaling $\zeta_{p}=p / 2$ and in a range of scales that may correspond to an inverse cascade of energy). The values of $\zeta_{p}$ for run B contrast with results obtained for the scaling exponents in nonhelical rotating turbulence from DNS for the direct cascade ${ }^{10,43}$ and from laboratory experiments for decaying flows, ${ }^{9}$ where a reduction of the intermittency was observed but anomalous scaling as a signature of intermittency was still present; as an example, in Ref. 43, for a nonhelical flow at late times with $\mathrm{Ro} \approx 0.07$ it was found that $\mu=0.24 \pm 0.02$. The possibility that the different behaviors reported in the experiments can be ascribed to helicity is tantalizing.

In the case of the helicity exponents $\xi_{p}$, the highest orders could not be measured for the reasons discussed above: the higher order dependence on the fields of the helical structure functions and the associated stronger fluctuations observed. However, results for run A are in agreement with previous studies of the direct cascade of helicity in isotropic and homogeneous turbulence, with the helicity being more intermittent than the velocity field (note, however, differences in the definitions used here and in the analysis in Ref. 25). This is illustrated by the smaller values of $\xi_{p}$ when compared with $\zeta_{p}$ in run A (see Fig. 7 and Table I). On the other hand, results in run B at low Rossby number seem to be of a different nature. The $\xi_{p}$ exponents up to $p=4$ are within error bars consistent with a linear (self-similar) scaling $\xi_{p} \approx 0.73 p$ (i.e., with a slightly larger slope than for $\zeta_{p}$ in the same run, see Fig. 7), but $\xi_{5}$ departs from such scaling (see also Table I). This departure would indicate intermittency in the helicity, a property that will be confirmed in Sec. $\mathrm{V}$ studying the PDFs of the increments. 

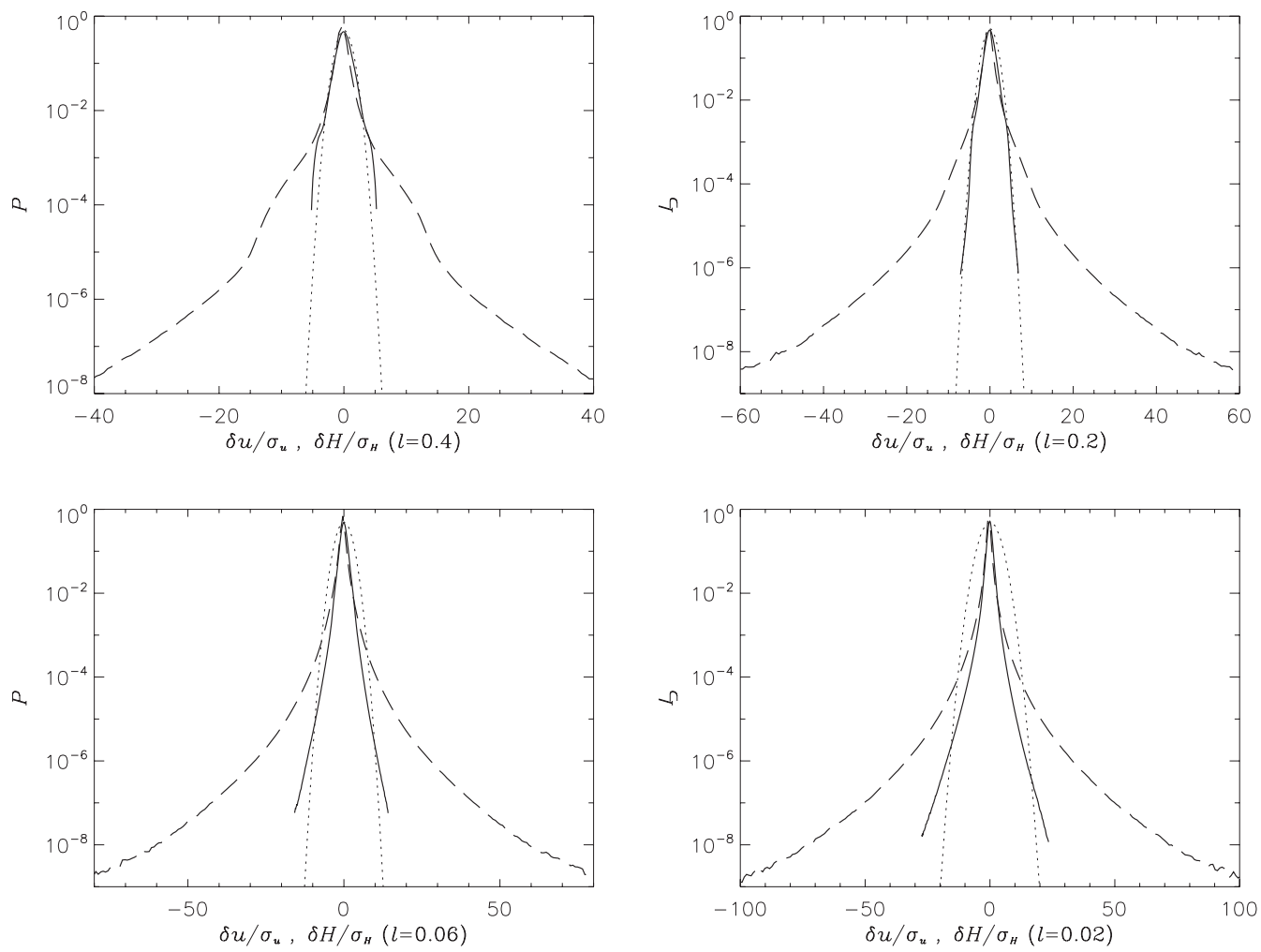

FIG. 8. PDFs at different intervals in the direct cascade for velocity (solid) and helicity (dashed) increments in the direction perpendicular to the axis of rotation. Increments are normalized by their variance. The dotted curve represents a Gaussian distribution with the same variance.

\section{PDFs}

The identification of multifractal (as opposed to scale independent) scaling in turbulent flows based on scaling exponents present several difficulties. It is well known that transients and finite size effects can give spurious multifractal scaling, ${ }^{44}$ and that logarithmic or subleading corrections to the spectrum can also lead to the same result. ${ }^{45}$ In this section we consider PDFs of the velocity and helicity increments. In a scale invariant flow, the velocity increments are expected to be Gaussian. On the other hand, in an intermittent flow, PDFs are expected to have strong non-Gaussian tails.

In Fig. 8 we show the PDFs of the velocity and helicity increments in the direction perpendicular to the axis of rotation for four values of $\ell_{\perp}=0.4,0.2,0.06$, and 0.02 . The PDFs are normalized by their variance, and a Gaussian with the same variance is shown as a reference. The PDFs of velocity increments for $\ell_{\perp}=0.4$ and 0.2 are close to Gaussian. Note that these increments correspond to scales in the anisotropic inertial range (see Figs. 3 and 4). PDFs close to Gaussian were also observed for $\delta u$ for other increments in this range. However, for $\ell_{\perp}=0.4$ a "bump" (which also decays as a Gaussian) can be observed in the tails. This bump disappears as smaller increments are considered and seems to be associated with the forcing (note that the mechanical forcing acts between $\ell \approx 0.78$ and 0.90$)$.

The bump is more evident in the PDF of helicity increments with $\ell_{\perp}=0.4$. For both values $\ell_{\perp}=0.4$ and 0.2 (in the direct cascade inertial range) the PDFs of helicity increments are different than for velocity increments: deviations from
Gaussianity are evident, and the PDFs show strong tails. The non-Gaussian tails are the signature of the presence of strong gradients, and of intermittency in the spatial distribution of helicity. These results confirm-independently of the scaling exponents analyzed in the preceding section-that the anisotropic direct cascade of energy toward smaller scales is close to Gaussian and scale independent, while the direct cascade of helicity is intermittent.

For increments in the range of scales where $S_{p}\left(\ell_{\perp}\right)$ is of the same order as $S_{p}\left(\ell_{\|}\right)$for $p \geq 4$ (see e.g., Fig. 4 ), both the PDFs of velocity and of helicity increments show nonGaussian tails (see Fig. 8 for $\ell_{\perp}=0.06$ and 0.02). This further confirms that at very small scales, the strongest events (in the tails of the PDFs) tend toward a recovery of isotropy, which would lead in turn to a traditional (and intermittent) direct cascade of energy. However, simulations at larger resolution and at different Reynolds and Rossby numbers will be required to verify if this recovery of isotropy occurs always near the dissipation range, or if a second isotropic inertial range develops when enough scale separation is available at scales smaller than the Ozmidov scale when rotation can presumably be neglected.

\section{STRUCTURES}

The results discussed in Secs. IV and V point toward an anisotropic and scale-invariant energy distribution in an intermediate range of scales (smaller than the forcing scale) and a highly intermittent helicity distribution in the same range of scales. In helical rotating turbulence, energy cascades both toward large and small scales, while helicity cas- 

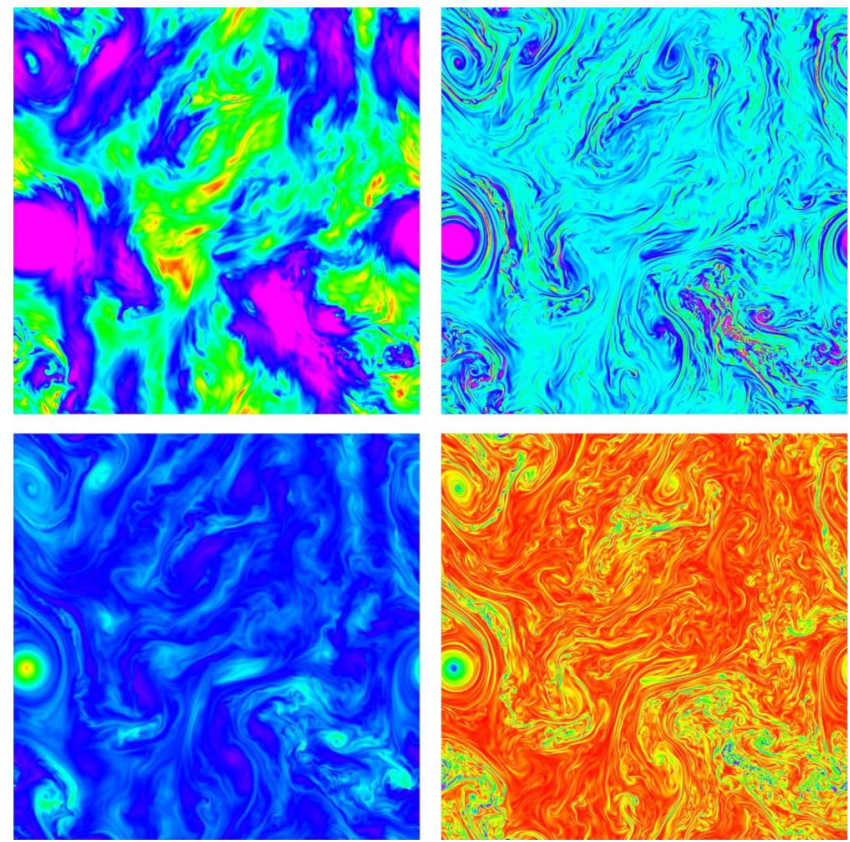

FIG. 9. (Color online) Slices of the energy density (top left), vorticity intensity (top right), $z$ component of the velocity (bottom left), and helicity density (bottom right) in run $\mathrm{B}$ at $t \approx 30$. Note the imprint of small scales in the vorticity and helicity (right column).

cades toward small scales dominating the direct cascade inertial range. In light of these facts, it is of interest to look at the structures that arise in the flow.

In Fig. 9 are displayed horizontal slices (in the plane perpendicular to the rotation axis) of the energy density, the vorticity intensity, the $z$ component of the velocity, and the helicity density in run B with Ro=0.06, at $t \approx 30$. While the energy density seems organized in large-scale patches (substantially larger than the forcing scale which corresponds roughly to $1 / 7$ of the box), the vorticity intensity and helicity density show small-scale structures. This is consistent with an inverse cascade of energy and a direct cascade dominated by the helicity. Note however that the energy density distribution in space is different from the distribution observed in two dimensional turbulence, where an inverse cascade of energy also takes place; in other words, the inverse cascade here differs from the purely two-dimensional case, be it only because the conservation of helicity (in the ideal case) induces the flow to keep some trace of three-dimensionality (and isotropy) at small scale, as noted before. Moreover, smooth structures at some intermediate scale can be observed in the helicity and the vorticity (see e.g., the left side of the box); these regions are also correlated with similar regions in the $z$ component of the velocity.

When run B is started from a previously isotropic state, the flow first becomes anisotropic and then a selforganization process starts that leads to the formation of columns. Those columns can be identified when the energy density, helicity density, or the vorticity intensity are visualized. The columns have strong $u_{z}$ (see e.g., Fig. 10). However, a few columns can be distinguished from the rest, in that they have a strong updraft velocity and concentrate in their core positive helicity, with strong relative helicity (strong align-
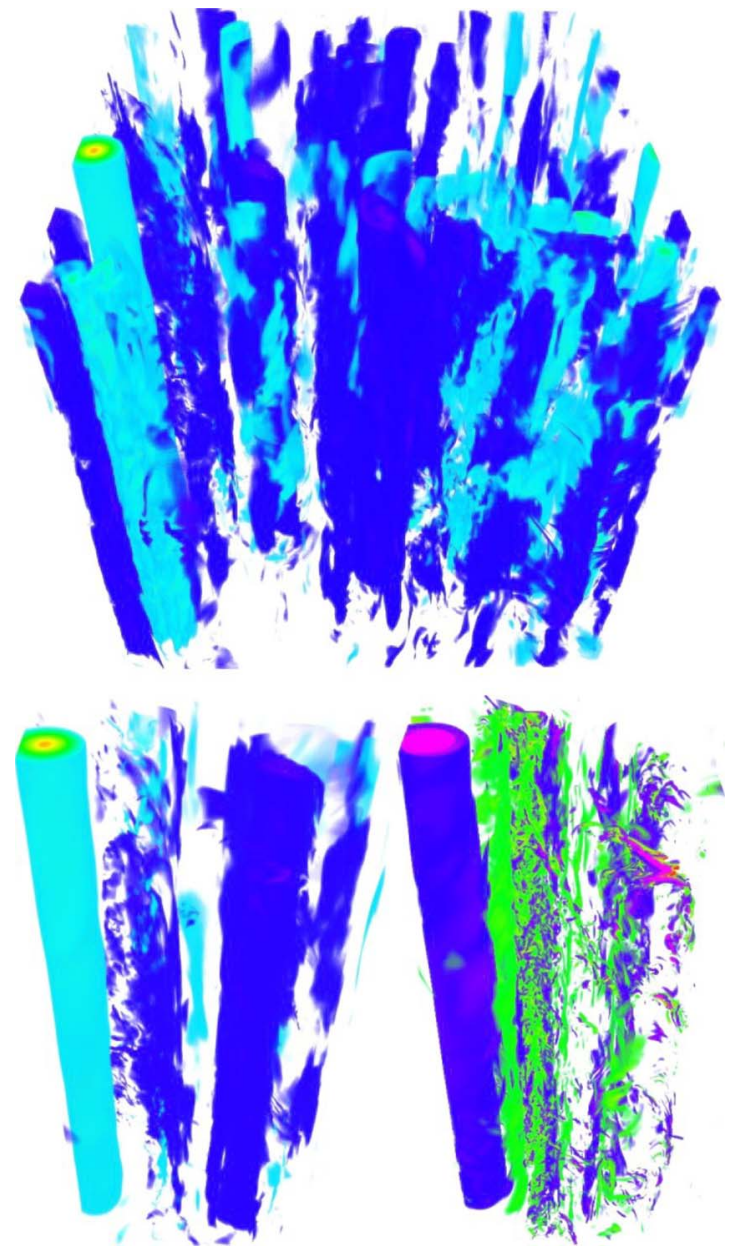

FIG. 10. (Color online) Three dimensional rendering of the $z$ component of the velocity in the entire domain in run $\mathrm{B}$ at $t \approx 30$ (above) and a zoom on a subregion (below) showing the $z$ component of the velocity in a columnlike structure (left) and its helicity density (right).

ment between velocity and vorticity). These columns are stable, and we were able to track these columns in the simulation for over ten turnover times.

Far from these structures, the flow displays a myriad of small scales, as illustrated by the small-scale filaments in the helicity density. These filaments are also organized in columnar structures, but the thick columns with net helicity live for much longer times. As a result, in real space the inverse cascade of energy can be identified as the system evolves in time and these helical structures merge with columns with a lesser amount of relative helicity, increasing the characteristic width of the column when its energy density is visualized, but keeping the thickness of the helical core in the column approximately constant. This is the result of the helicity injected in the flow cascading directly to smaller scales, which allows for a localized helical column, but prevents the formation of a thick distribution of helicity in a column filling all space. Accompanying the direct cascade, strong fluctuations of helicity are observed in the turbulent columns with the characteristic size of the vortex filaments. 


\section{CONCLUSIONS}

The analysis of the structure functions and of the PDFs of velocity and helicity increments from data stemming from DNS of helical rotating turbulence at high resolution, showed that, at least for the strongest events in the small scales, isotropy is recovered at sufficiently small scales. This is observed both in the collapse of the parallel and perpendicular structure functions, as well as in the transition from near-Gaussian statistics of the velocity increments in the inertial range toward PDFs with strong tails in the same range of scales as observed in the structure functions. More studies will be required to see if this transition takes place for all orders when the Reynolds number is large enough, leading to a sufficient scale separation.

Concerning scaling exponents, we also confirmed that in the anisotropic direct cascade range the velocity increments are (within error bars) scale invariant (i.e., nonintermittent) while helicity increments are intermittent. This is further confirmed by the PDFs, which show strong non-Gaussian tails for the helicity and are near Gaussian for velocity increments in the anisotropic range. The scaling exponents for the helicity are consistent (within error bars) with a scale invariant dependence $\xi_{p} \approx 0.73 p$ up to $p=4$, but $\xi_{5}$ departs from a straight line. More data will be required to confirm a possible bifractal or multifractal scaling for the helicity, although we would like to point out that a bifractal scaling would be consistent with the two types of helical structures observed in visualizations of the flow (the large-scale laminar columns and the small-scale vortex filaments), and reminiscent of the behavior or other systems with two type of structures, e.g., the Burgers equation, which develops smooth ramps connected by sharp shocks.

\section{ACKNOWLEDGMENTS}

Computer time was provided by NCAR. NCAR is sponsored by the National Science Foundation. P.D.M. acknowledges support from Grant Nos. UBACYT X468/08 and PICT-2007-02211, and from the Carrera del Investigador Científico of CONICET.

${ }^{1}$ U. Frisch, Turbulence: The Legacy of A. N. Kolmogorov (Cambridge University Press, Cambridge, 1995).

${ }^{2}$ K. R. Sreenivasan and R. A. Antonia, "The phenomenology of small-scale turbulence," Annu. Rev. Fluid Mech. 29, 435 (1997).

${ }^{3}$ L. Sorriso-Valvo, V. Carbone, P. Veltri, H. Politano, and A. Pouquet, "Non-Gaussian probability distribution functions in two-dimensional magnetohydrodynamic turbulence," Europhys. Lett. 51, 520 (2000).

${ }^{4}$ P. D. Mininni and A. Pouquet, "Energy spectra stemming from interactions of Alfvén waves and turbulent eddies," Phys. Rev. Lett. 99, 254502 (2007).

${ }^{5}$ D. Bernard, G. Boffetta, A. Celani, and G. Falkovich, "Conformal invariance in two-dimensional turbulence," Nat. Phys. 2, 124 (2006).

${ }^{6} \mathrm{C}$. Cambon and L. Jacquin, "Spectral approach to non-isotropic turbulence subjected to rotation," J. Fluid Mech. 202, 295 (1989).

${ }^{7}$ F. Waleffe, "Inertial transfers in the helical decomposition," Phys. Fluids A 5, 677 (1993).

${ }^{8}$ L. M. Smith and Y. Lee, "On near resonances and symmetry breaking in forced rotating flows at moderate Rossby number," J. Fluid Mech. 535, 111 (2005)

${ }^{9}$ J. Seiwert, C. Morize, and F. Moisy, "On the decrease of intermittency in decaying rotating turbulence," Phys. Fluids 20, 071702 (2008).
${ }^{10}$ W.-C. Müller and M. Thiele, "Scaling and energy transfer in rotating turbulence," Europhys. Lett. 77, 34003 (2007).

${ }^{11}$ P. D. Mininni, A. Alexakis, and A. Pouquet, "Scale interactions and scaling laws in rotating flows at moderate Rossby numbers and large Reynolds numbers," Phys. Fluids 21, 015108 (2009).

${ }^{12}$ C. N. Baroud, B. B. Plapp, H. L. Swinney, and Z.-S. She, "Scaling in three-dimensional and quasi-two-dimensional rotating turbulent flows," Phys. Fluids 15, 2091 (2003)

${ }^{13} \mathrm{P}$. Hoyng, "Helicity fluctuations in mean field theory: an explanation for the variability of the solar cycle?" Astron. Astrophys. 272, 321 (1993).

${ }^{14} \mathrm{P}$. Charbonneau, "Multiperiodicity, chaos, and intermittency in a reduced model of the solar cycle," Sol. Phys. 199, 385 (2001).

${ }^{15}$ P. D. Mininni and D. O. Gómez, "A new technique for comparing solar dynamo models and observations," Astron. Astrophys. 426, 1065 (2004).

${ }^{16}$ J. R. Kulkarni, L. K. Sadani, and B. S. Murthy, "Wavelet analysis of intermittent turbulent transport in the atmospheric surface layer over a monsoon trough region," Boundary-Layer Meteorol. 90, 217 (1999).

${ }^{17}$ P. Mininni and A. Pouquet, "Rotating helical turbulence. I. Global evolution and spectral behavior," Phys. Fluids 22, 035105 (2010).

${ }^{18} \mathrm{D}$. K. Lilly, "The structure, energetics, and propagation of rotating convective storms. Part II: Helicity and storm stabilization," J. Atmos. Sci. 43, 126 (1986).

${ }^{19}$ B. W. Kerr and G. L. Darkow, "Storm-relative winds and helicity in the tornadic thunderstorm environment," Weather Forecast. 11, 489 (1996).

${ }^{20}$ P. M. Markowski, J. M. Straka, E. N. Rasmussen, and D. O. Blanchard, "Variability of storm-relative helicity during VORTEX," Mon. Weather Rev. 126, 2959 (1998).

${ }^{21}$ P. D. Mininni and A. Pouquet, "Helicity cascades in rotating turbulence," Phys. Rev. E 79, 026304 (2009).

${ }^{22}$ E. Akylas, S. C. Kassinos, and C. A. Langer, "Rapid shear of initially anisotropic turbulence in a rotating frame," Phys. Fluids 19, 025102 (2007).

${ }^{23}$ A. N. Kolmogorov, "Dissipation of energy in locally isotropic turbulence," Dokl. Akad. Nauk SSSR 32, 16 (1941).

${ }^{24}$ Q. Chen, S. Chen, and G. L. Eyink, "The joint cascade of energy and helicity in three-dimensional turbulence," Phys. Fluids 15, 361 (2003).

${ }^{25}$ Q. Chen, S. Chen, G. L. Eyink, and D. D. Holm, "Intermittency in the joint cascade of energy and helicity," Phys. Rev. Lett. 90, 214503 (2003).

${ }^{26}$ O. G. Chkhetiani, "On the third-moments in helical turbulence," JETP Lett. 63, 808 (1996).

${ }^{27} \mathrm{~S}$. Kurien, "The reflection-antisymmetric counterpart of the KármánHowarth dynamical equation," Physica D 175, 167 (2003).

${ }^{28}$ T. Gomez, H. Politano, and A. Pouquet, "Exact relationship for third-order structure functions in helical flows," Phys. Rev. E 61, 5321 (2000).

${ }^{29} \mathrm{~A}$. Tsinober, E. Kit, and T. Dracos, "Experimental investigation of the field of velocity gradients in turbulent flows," J. Fluid Mech. 242, 169 (1992).

${ }^{30}$ L. Jacquin, O. Leuchter, C. Cambon, and J. Mathieu, "Homogeneous turbulence in the presence of rotation," J. Fluid Mech. 220, 1 (1990).

${ }^{31}$ C. Morize, F. Moisy, and M. Rabaud, "Decaying grid-generated turbulence in a rotating tank," Phys. Fluids 17, 095105 (2005).

${ }^{32}$ P. J. Staplehurst, P. A. Davidson, and S. B. Dalziel, "Structure formation in homogeneous freely decaying rotating turbulence," J. Fluid Mech. 598, 81 (2008).

${ }^{33}$ L. M. Smith and F. Waleffe, "Transfer of energy to two-dimensional large scales in forced, rotating three-dimensional turbulence," Phys. Fluids 11, 1608 (1999).

${ }^{34}$ C. Cambon, N. N. Mansour, and F. S. Godeferd, "Energy transfer in rotating turbulence," J. Fluid Mech. 337, 303 (1997).

${ }^{35}$ P. A. Davidson, P. J. Staplehurst, and S. B. Dalziel, "On the evolution of eddies in a rapidly rotating system," J. Fluid Mech. 557, 135 (2006).

${ }^{36}$ I. Arad, B. Dhruva, and S. Kurien, V. S. L'vov, I. Procaccia, and K. R. Sreenivasan, "Extraction of anisotropic contributions in turbulent flows," Phys. Rev. Lett. 81, 5330 (1998).

${ }^{37} \mathrm{~L}$. Biferale and I. Procaccia, "Anisotropy in turbulent flows and in turbulent transport," Phys. Rep. 414, 43 (2005).

${ }^{38}$ M. A. Taylor, S. Kurien, and G. L. Eyink, "Recovering isotropic statistics in turbulence simulations: The Kolmogorov 4/5th law," Phys. Rev. E 68, 026310 (2003)

${ }^{39}$ B. Dubrulle and L. Valdettaro, "Consequences of rotation in energetics of accretion disks," Astron. Astrophys. 263, 387 (1992).

${ }^{40} \mathrm{O}$. Zeman, "A note on the spectra and decay of rotating homogeneous turbulence," Phys. Fluids 6, 3221 (1994).

${ }^{41}$ Y. Zhou, "A phenomenological treatment of rotating turbulence," Phys. Fluids 7, 2092 (1995). 
${ }^{42}$ T. Gotoh, D. Fukayama, and T. Nakano, "Velocity field statistics in homogeneous steady turbulence obtained using a high-resolution direct numerical simulation," Phys. Fluids 14, 1065 (2002).

${ }^{43}$ P. D. Mininni, A. Alexakis, and A. Pouquet, "Nonlocal interactions in hydrodynamic turbulence at high Reynolds numbers: The slow emergence of scaling laws," Phys. Rev. E 77, 036306 (2008).
${ }^{44}$ E. Aurell, U. Frisch, A. Noullez, and M. Blank, "Bifractality of the devil's staircase appearing in the Burgers equation with brownian initial velocity," J. Stat. Phys. 88, 1151 (1996).

${ }^{45}$ D. Mitra, J. Bec, R. Pandit, and U. Frisch, "Is multiscaling an artifact in the stochastically forced Burgers equation?" Phys. Rev. Lett. 94, 194501 (2005). 